\title{
A big bite of the past
}

By standing up for themselves between 3 million and 4 million years ago, Lucy and her fellow Australopithecus afarensis caused quite a stir. But bipedalism is just one factor in the rich mix of human evolution, as amply shown in the revised, updated and expanded From Lucy to Language (Simon \& Schuster, \$65).

Donald Johanson, who discovered Lucy, and his co-writer Blake Edgar have added the big finds since 1996 to their brilliant overview, including the Indonesian 'hobbit' Homo floresiensis. And as this snap of $A$. afarensis teeth from Ethiopia reveals, the expanded range of photos - many at actual size - remain jaw-droppingly spectacular.

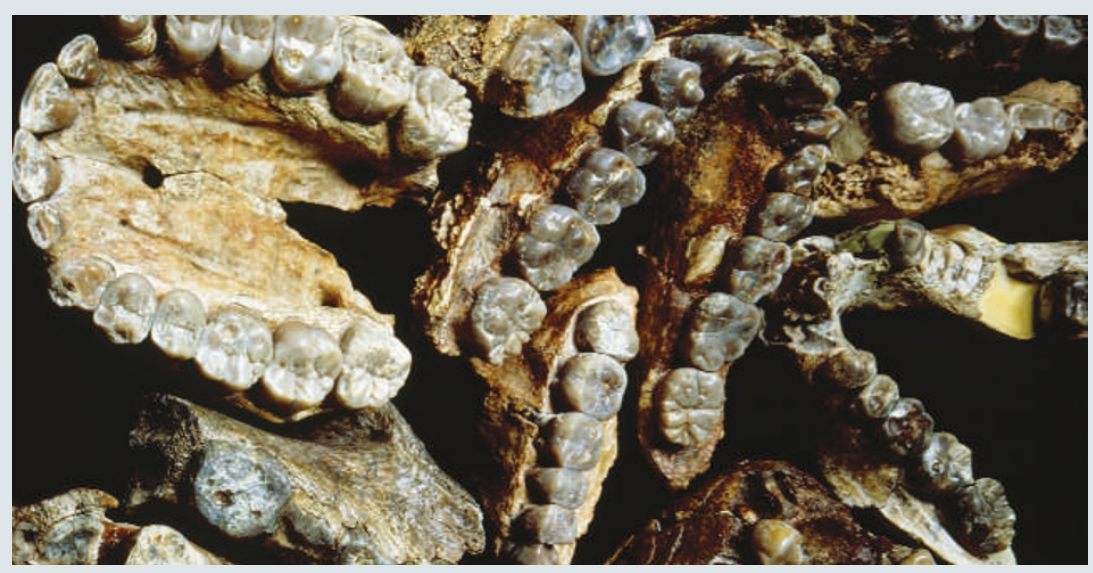

\section{Back to basics}

\section{Darwinian Reductionism: Or, How to Stop Worrying and Love Molecular Biology by Alex Rosenberg \\ University of Chicago Press: 2006. 272 pp. $\$ 40, \pm 25.50$}

\section{Bruce H. Weber}

The understanding we have gained about the molecular basis of living systems and their processes was a triumph of twentieth-century science. Since the structure of DNA was elucidated in 1953, molecular biologists have been deepening our insights into a wide range of biological phenomena. It has been a heady time: it seemed that mendelian genetics would be reduced to the macromolecular chemistry of nucleic acids, with biology set to become a mature science in the same way as physics and chemistry. The emerging field of the philosophy of biology inherited the reductionist framework of logical empiricism. But as our knowledge of molecular biology deepened, many philosophers of biology, including David Hull, Philip Kitcher, Eliot Sober, Evelyn FoxKeller and Paul Griffiths, saw that the reductionist approach faced serious problems.

There is no simple correlation between the mendelian gene and the increasingly complex picture provided by molecular genetics. To make matters worse, the theory to be reduced was presumably the population-genetic version of darwinian natural selection, which had from the start excluded phenomena about development and their possible link to evolutionary dynamics. Given this absence, Ernst Mayr, a founder of the modern evolutionary synthesis, argued that, although biological systems did not violate the laws of chemistry and physics, evolving biological systems have properties that cannot be reduced to such laws. The crux of the issue as Mayr saw it was that, whereas the physical sciences deal only in proximate explanations, the biological sciences also deal with ultimate explanations relating to evolutionary descent and the action of selection to produce adapted function. This, Mayr argued, resulted in the autonomy of biology with respect to the physical sciences. Alex Rosenberg's book Darwinian Reductionism is a response to the anti-reductionist position in contemporary philosophy of biology and to the autonomist stance of some biologists.

Rosenberg's thesis is that biological phenomena, including their functional aspects, are best understood at the level of their macromolecular constituents and their interactions in cellular environments that are themselves made up of other molecules. This has been, and continues to be, he argues, a successful, progressive research programme. He focuses in particular on the great advances in our understanding of developmental molecular biology, which teaches us how the genes that are involved in development function, interact and work with chemical gradients, for example, to produce morphology. Rosenberg provides an accessible review of current ideas on the 'wiring' of such gene complexes and the way they help account for morphological evolution. He is one of the first philosophers to consider the implications of 'evo-devo' (evolutionary developmental biology), and seizes the opportunity to promote a reductionist interpretation that was simply not possible with population genetics.

He shows a good grasp of the scientific details of developmental molecular biology, but it is unfortunate that in the introduction he gets the molecular details of sickle-cell anaemia wrong and then describes a resulting arterial blockage, rather than the lysis of red blood cells. This should not have survived the reviewing and editing process, but it is the only serious lapse. When he returns to the issue of mutant haemoglobins later in the book, he gets the molecular details for sickle-cell haemoglobin correct.

To bridge Mayr's gap between ultimate (natural selection) causes and proximate (structural and functional) causes, Rosenberg cites Theodosius Dobzhansky's dictum that nothing in biology makes sense except in the light of evolution. The various molecules in cells and the gene sequences of the macromolecules are products of previous selection by which their proximately causal (structural and functional) properties were screened. In bringing causality to bear on explanation, he makes use of the distinction between 'how possible' and 'why necessary' explanations. Ultimate historical explanations of current biological structures and functions are 'how possible' in type. But why particular molecular arrangements were selected in the past has the force of 'why necessary' explanation. This removes the burden from selectional dynamics of having to be predictive in order to be reductionist.

Rosenberg realizes that theory reductionism requires the theory of darwinian natural selection to be grounded in, or reduced to, a principle of natural selection at the level of chemical systems in which both stability and replicability are selected for. In effect, he produces a scenario in which biological selection can be reduced to chemical selection during the origin of life. This crucial move needs more careful analysis than Rosenberg provides. He gives, in effect, a 'how possible' explanation for the emergence of life and biological selection, but not a 'why necessary' one. For that he would need to deal with the literature of the origin of life and the more general recent work on complexity. Such an investigation would show that phenomena in these areas are more emergent than Rosenberg believes, and that there is a need to develop a theory of organization and emergence. Research on emergent complexity is still a work in progress, but it may undercut Rosenberg's thesis by providing a fully naturalistic, non-reductionist account of emergence. Such a non-reductionist account would not be anti-reductionist in the sense Rosenberg uses the term, but would offer a 'why necessary' explanation of the emergent phenomena. Bruce H. Weber is emeritus professor in the Department of Chemistry and Biochemistry, California State University, Fullerton, and in the Division of Science and Natural Philosophy, Bennington College, Bennington, Vermont, USA. 